\title{
Determination and Dietary Intake Risk Assessment of 35 Pesticide Residues in Cowpea (Vigna Unguiculata L. Walp) from Hainan Province, China
}

Qun Zhang ( $\nabla$ zhangqun123@zju.edu.cn )

Chinese Academy of Tropical Agricultural Sciences

Chen Ma

Chinese Academy of Tropical Agricultural Sciences

Yun Duan

Chinese Academy of Tropical Agricultural Sciences

Xiaopeng Wu

Chinese Academy of Tropical Agricultural Sciences

Daizhu Lv

Chinese Academy of Tropical Agricultural Sciences

Jinhui Luo

Chinese Academy of Tropical Agricultural Sciences

\section{Research Article}

Keywords: pesticide residue, cowpea (Vigna unguiculata L. Walp), GC-MS/MS, risk assessment

Posted Date: November 17th, 2021

DOI: https://doi.org/10.21203/rs.3.rs-1037696/v1

License: () (7) This work is licensed under a Creative Commons Attribution 4.0 International License. Read Full License

Version of Record: A version of this preprint was published at Scientific Reports on April 1st, 2022. See the published version at https://doi.org/10.1038/s41598-022-09461-w. 


\section{Abstract}

The presence of pesticide residues in cowpea raises serious health concerns. In this study, a novel, sensitive, high-performance method was developed to simultaneously analyse the residues of 35 pesticides in cowpea samples from growing areas in Hainan provinces of China, from November in 2018 and June in 2021, using modified QuEChERS sample pretreatment coupled with gas chromatography-tandem mass spectrometry (GC-MS/MS). The limits of detection (LOD) and quantification (LOQ) values of 35 pesticides in the cowpea matrix ranged from $0.3 \mu \mathrm{g} / \mathrm{kg}$ to $2.5 \mu \mathrm{g} / \mathrm{kg}$ and $1.0 \mu \mathrm{g} / \mathrm{kg}$ to $8.0 \mu \mathrm{g} / \mathrm{kg}$, respectively. Twenty-seven different pesticides were detected, and twelve of them were banned pesticides on legumes in China. Residues for ten pesticides in $17.1 \%$ of the samples exceeded their MRLs with the highest exceedance of $380 \%, 80.8 \%$ of samples contained one or more pesticide residues, and the most frequently detected pesticide was chlorfenapyr with a detection rate of $46.3 \%$. In addition, the pesticide triazophos was detected through different years and regions. Furthermore, the chronic dietary exposure risk (\%ADI) of the detected pesticides showed less than $100 \%$ in Chinese people of different age groups, which was evaluated by the national estimated acceptable daily intake.

\section{Introduction}

Cowpea, Vigna unguiculata (Linn.) Walp, also known as bean, long bean, etc., is a kind of vegetable with high nutritional value, which has an important position and role in China's northern vegetable industry ${ }^{1}$. Hainan province is the main cowpea growing area of China's northern vegetable industry, with the yearly cultivation area of cowpea around $2.0 * 10^{4}$ hectare, and the annual output of cowpea more than $5.0^{*} 10^{6}$ tons from Statistical Bureau of Hainan Province in $2020^{2}$. The high temperature and humidity environment in Hainan province is conducive to the occurrence of diseases and insect pests ${ }^{3}$. In addition, it is well known cowpea is a flower and fruit crop at the same time, which means its flowering period is also the harvest time $\mathrm{e}^{4}$. Thus, there is a higher incidence of pest and disease outbreaks on cowpeas. Therefore, farmers often spray a large number of pesticides to improve the yield of cowpea, and the more common pesticides used were fipronil, cyfluthrin, cyhalothrin, cypermethrin, pyridaben, pyrimethanil and so on. In this study, 35 pesticides including prohibited pesticides, easy to exceed conventional pesticides, and commonly used pesticides in vegetable in China, were considered. Some of these pesticides can occur in the samples by legal application to the crops and others by illegal application.

Due to the widespread use of these pesticides, there are more opportunities for residues from the environment to enter the human body through food. In recent years, multi-residue methods have been used for detecting pesticide residues at trace concentrations in vegetables, fruits and other food products, including gas chromatography (GC $)^{5-9}$, liquid chromatography (LC) ${ }^{10-12}$, spectral analysis ${ }^{13}$, immunoanalysis ${ }^{14}$ and electrochemical sensor technology ${ }^{15}$, 16 , and so on. The GC or LC coupled to tandem mass spectrometry (MS/MS) has been used for accurately simultaneous determination of pesticide residues in agricultural and animal products ${ }^{17-20}$. Pesticide extraction in agricultural and animal products has been carried out using many different extraction techniques: solidphase extraction $(\mathrm{SPE})^{8,21,22}$, magnetic solid-phase extraction (MSPE) ${ }^{23,24}$; dispersive solid-phase extraction $(\mathrm{DSPE})^{17,25,26}$, solid-phase micro-extraction $(\mathrm{SPME})^{27-29}$, liquid-solid extraction (LSE) ${ }^{22}$, accelerated solvent extraction (ASE) ${ }^{30}$, ultrasonic assisted extraction (UAE) ${ }^{31}$, and the quick easy cheap effective rugged and safe method (QuEChERS) ${ }^{26,32,33}$. Within these techniques, the QuEChERS method is becoming more popular in recent years due to its simplicity, fast, low-cost and high throughput, and minimal solvent requirement ${ }^{26,32,33-36}$

The aims of this study were (1) to establish a rapid analysis method for the determination of 35 pesticides in cowpea by QuEChERS-gas chromatographytandem mass spectrometry (QuEChERS-GC-MS/MS); (2) to analyze residue levels of 35 pesticides in cowpea samples from Hainan province in China; and (3) to preliminary assess the chronic dietary intake risk of pesticides detected in cowpea by different populations.

\section{Materials And Methods}

\section{Reagents and Materials}

Thirty-five pesticide residues were detected, including acephate, azoxystrobin, chlordimeform, chlorfenapyr, chlorpyrifos-ethyl, coumaphos, cyfluthrin, cyhalothrin, cypermethrin, dicofol, difenoconazole, dimethoate, endosulfan, ethoprophos, fenitrothion, fenpropathrin, fenvalerate, fipronil, fluvalinate, Isazofos, isocarbophos, isofenphos-methyl, malathion, methamidophos, omethoate, parathion (ethyl), parathion-methyl, pendimethalin, phorate, profenofos, pyridaben, pyrimethanil, sulfotep, terbufos, triazophos.

Individual pesticide analytical standards were purchased from were purchased from Dr. Ehrenstorfer GmbH (Germany), and stored in a freezer at -20 ${ }^{\circ} \mathrm{C}$. Acetonitrile and n-hexane (HPLC grade) was purchased from Fisher Scientific (USA). The stock standard solution was prepared separately in a concentration around $1000 \mathrm{mg} / \mathrm{L}$, using $\mathrm{n}$-hexane as solvent. Mixed working standard solutions at a series of concentrations were prepared by the dilution of aliquots of the stock solution in n-hexanes ${ }^{19-21}$. The standard working solution was used to prepare matrix-matched standards and to spike samples in the validation study. The stock and working standard solutions were stored in amber glassware under appropriate conditions, i.e., at $-20{ }^{\circ} \mathrm{C}$ until the time of analysis.

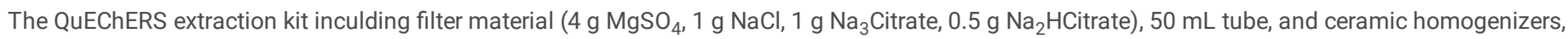
were purchased from Agilent Technologies (Part Number: 5982-5650CH). The QuEChERS dispersive kit containing 2 mL tube with 25 mg PSA, 2.5 mg GCB, $150 \mathrm{mg} \mathrm{MgSO}$, were purchased from Agilent Technologies (Part Number: 5982-5221).

A total of 574 samples of cowpea (Vigna unguiculata L. Walp) were purchased through growing areas (Sanya, Ledong, Lingshui, Wanning, Chengmai, Haikou, etc) in Hainan provinces of China, from November in 2018 and June in 2021. At least $3 \mathrm{~kg}$ of cowpea pods for each sample were bought and sealed in a sterile polyethylene bag with a unique identification mark by NY/T 762-2004 ${ }^{37}$. After collection, each sample was homogenized within $8 \mathrm{~h}$ and stored at $-20{ }^{\circ} \mathrm{C}$ until analyzing. 
The pesticides were analyzed using an Thermo Scientific ${ }^{\text {TM }}$ Trace 1310-TSQ 9000. An TG-5SILMS glass capillary column (length $30 \mathrm{~m}$, internal diameter $0.25 \mathrm{~mm}$, and film thickness $0.25 \mu \mathrm{m}$, Thermo) was used for the separation. The GC program was as follows: total run time of $23.5 \mathrm{~min}$, the column was held initially at a temperature of $70{ }^{\circ} \mathrm{C}$ and held for $1 \mathrm{~min}$, increased at $25^{\circ} \mathrm{C} / \mathrm{min}$ to $150^{\circ} \mathrm{C}$ and held for $3 \mathrm{~min}$, increased at $15^{\circ} \mathrm{C} / \mathrm{min}$ to $200{ }^{\circ} \mathrm{C}$ and held for $3 \mathrm{~min}$, increased at $20^{\circ} \mathrm{C} / \mathrm{min}$ to $300^{\circ} \mathrm{C}$ and held for $5 \mathrm{~min}$. The temperatures corresponding to the transfer line and the ion trap were all $300{ }^{\circ} \mathrm{C}$, respectively, and the ionisation energy was $70 \mathrm{eV}$. The injection port temperature was $260^{\circ} \mathrm{C}$, and $1-\mu \mathrm{L}$ samples were injected splitless. Helium was used as a carrier at a flow rate of $1.2 \mathrm{~mL} / \mathrm{min}$. The mass spectrometer was operated in selected reaction monitoring (SRM) mode, as listed in Table 1. The solvent delay was set at $4 \mathrm{~min}$.

\section{Sample preparation and extraction}

A QuEChERS method was chosen for the sample preparation in the initial experiment ${ }^{32-36}$. Briefly, a $10 \mathrm{~g}$ (accurate to $0.01 \mathrm{~g}$ ) portion of milled sample was added to a $50 \mathrm{~mL}$ polytetrafluoroethylene (PTFE) centrifuge tube. Then, $10 \mathrm{~mL}$ of acetonitrile was added and the samples were homogenized for 2 min. After that, filter materia were added and the samples were vigorously shaken for $1 \mathrm{~min}$. The extract was then centrifuged (10000 rpm) for $5 \mathrm{~min}$. Afterwards, $1.5 \mathrm{~mL}$ of the supernatant (acetonitrile phase) was transferred to a $2 \mathrm{~mL}$ centrifuge tube containing $25 \mathrm{mg} \mathrm{PSA}, 2.5 \mathrm{mg} \mathrm{GCB}, 150 \mathrm{mg} \mathrm{MgSO}{ }_{4}$, which was then vigorously shaken again for $1 \mathrm{~min}$. The tube was then centrifuged again (12000 rpm) for $5 \mathrm{~min}$. Finally, the acetonitrile extracts were filtered through a 0.22 $\mu \mathrm{m}$ PTFE filter (Milford, Boston, MA, USA), and analyzed by GC-MS/MS.

\section{Method accuracy}

The method accuracy was performed on 5 replicates on cowpea at each of the three spiking levels, 50, $100 \mathrm{and} 250 \mu \mathrm{g} / \mathrm{kg}$. The limits of detection (LOD) were considered the lowest analytical concentrations that yielded a signal-to-noise ratio (S/N) of 3, and the limits of detection quantification ( $\mathrm{LOQ}$ ) were set at the minimum concentration that could be quantified with acceptable values of recovery (70 120\%) and relative standard deviation (RSD $\leq 20 \%$ ), as advised by the European Union SANTE/12682/2019 regulatory guidelines ${ }^{38}$. The linearity of the matrix and solvent standard curves were injected at $2.5,5$, $10,50,100,250$ and $500 \mu \mathrm{g} / \mathrm{L}$ concentrations. The matrix effect (ME) was assessed by comparing the slopes of seven-point matrix-matched calibration curves with the slopes of calibration curves in the solvent.

\section{Dietary intake risk assessment}

The chronic dietary exposure risk (\%ADI) of pesticide residues in people of different age groups were calculated as follows:

$$
\% A D I=\frac{C \times F}{b w \times A D I} \times 100
$$

Where \%ADI is the chronic exposure risk ${ }^{39}, \mathrm{~F}(\mathrm{~kg})$ is the average daily intake of a certain food in China (Table 2), bw is the average body weight of the Chinese of different age groups (Table 2), $C_{i}(\mathrm{mg} / \mathrm{kg})$ is the average concentration of pesticide residues in cowpea of Hainan province, China (Table 3), and ADI $(\mathrm{mg} / \mathrm{kg} \cdot \mathrm{bw})$ is the acceptable daily intake of detectable pesticide residues (Table 3 ). When \%ADK 100 , the risk is acceptable and does not constitute a health threat in the long term, when $\% A D I$ is higher than 100 , it poses an unacceptable risk ${ }^{39}$.

\section{Results And Discussion}

\section{Matix effects and method accuracy}

The complexity of the vegetable matrix may have some effect on the analysis, and may inhibit or enhance the response, thus affecting the accuracy, selectivity and sensitivity of the method ${ }^{35}, 36$. If more than $20 \%$ signal suppression or enhancement, the matix effect (ME) should to be addressed in calibration $^{38}$. In this study, $11.4 \%$ of the thirty-five pesticides showed negligible $\mathrm{ME}(\mathrm{ME}<20 \%)$, and $48.6 \%$ of them showed medium ME $(20 \%<\mathrm{ME}<50 \%)$, while $40.0 \%$ of them suffered strong signal suppression (ME > 50\%) (Table 4). It has been reported that $98 \%$ of the total compounds analyzed by GC-MS/MS presented significant enhancement caused by the co-extraction of matrix components ${ }^{36}$. On the contrary, only $7 \%$ of pesticides showed signal suppression in complex herb matrices ${ }^{40}$. According to Krynitsky et al., even after comprehensive extensive sample extracts, there were still sufficient co-extraction compounds that could result in signal inhibition or signal enhancement, affecting quantity analysis adversely ${ }^{41}$. Therefore, to avoid the ME, the results were quantified by external standard method with matrix standard solution.

The results of method accuracy by external standard method with matrix standard solution are listed in Table 4 . It showed that the average recoveries of 35 pesticides were $83.2 \% \varangle 116.5 \%$ when the spiked levels were 50,100 , and $250 \mu \mathrm{g} / \mathrm{kg}$, with relative standard deviations (RSDs) in the range of $0.8 \% \otimes 9.8 \%$ $(n=5)$. The calibration curves of 35 pesticides were from $2.5 \mu \mathrm{g} / \mathrm{L}$ to $500 \mu \mathrm{g} / \mathrm{L}$, and the correlation coefficients were all greater than 0.9990 . In addition, the LODs were ranged from 0.3 to $2.5 \mu \mathrm{g} / \mathrm{kg}$, and the LOQs were ranged from 1.0 to $8.0 \mu \mathrm{g} / \mathrm{kg}$, which were lower than the Chinese MRLs (Table 3 ). According to the Guidance SANTE/12682/201938, this method can meet the requirements for determination of the selected pesticides in cowpea sample.

\section{Verification and analysis of cowpea samples}

The validated analytical method was used to analyse 35 pesticide residues in 574 cowpea samples collected from market, super-market and planting bases from hainan province, China. As shown in Fig 1, 27 of the 35 pesticides were detected at least once. There were 8 pesticides with detection rate above $10 \%$. 
The most frequently detected pesticide was the insecticide chlorfenapyr (46.3\%), followed by the fungicide difenoconazole (39.9\%), insecticide cypermethrin (36.8\%), acaricide pyridaben (19.7\%), then by insecticide profenofos (18.1\%), chlorpyrifos-ethyl (14.5\%), cyhalothrin (12.0\%) and fenpropathrin (11.0\%). According to the prohibited pesticides on legumes in China, twelve banned pesticides, acephate, chlordimeform, chlorpyrifos-ethyl, coumaphos, dicofol, fipronil, isazofos, isocarbophos, methamidophos, parathion-methyl, sulfotep, and triazophos, were found in $2.8 \%, 5.9 \%, 14.5 \%, 0.5 \%, 0.7 \%, 5.6 \%, 5.4 \%, 0.9 \%$, $1.2 \%, 3.8 \%, 0.2 \%$ and $3.8 \%$ of the total samples, respectively. Furthermore, of the 27 detected pesticide residues, the maximum residue limits (MRLs) priority referenced in GB/T 2763-202142, followed by in Chinese regulations (the MRLs of pesticide in vegetable routine monitoring in 2015) ${ }^{43}$, the list of prohibited pesticides on legumes in China ${ }^{44}$, and the MRLs of pesticide in European Commission ${ }^{45}$, which shown in Table 3 . It showed that the residues for ten pesticides in $17.1 \%$ of the samples exceeded their MRLs with the highest exceedance of $380 \%$. In addition, the MRLs exceedance rates were found for cypermethrin (8.5\%), difenoconazole (6.4\%), parathion-methyl (3.8\%), chlorfenapyr (3.1\%), cyfluthrin (1.4\%), cyhalothrin (1.4\%), pyridaben (0.9\%), fenvalerate $(0.3 \%)$, fenitrothion $(0.2 \%)$ and fenpropathrin $(0.2 \%)$, respectivly. This implied that these frequently detected pesticides were used widely and extensively in the cultivation of cowpea in Hainan provice, China. For the production and safe supply of agricultural products, the goverment need to strengthen oversight over the agricultural supplies market, strictly control the sale and use of prohibited pesticides, and strengthen the training and management of sales staff in agricultural stores. It also suggested that the rational use of these pesticides should be regulated.

As shown in Fig 2, samples with multiple pesticide residues (two or more detected pesticide residues) accounted for $59.5 \%$ of the total number of samples, samples contained one pesticide for $21.3 \%$, and residue-free samples for $19.2 \%$. The overall rate of multiple residues samples was higher than the rate of samples with no residue and a single residue, and sample numbers decreased with the increase of pesticide residues. This finding is consistent with those previous studies in cowpea, green pepper, cucumber, peach and apple $1,3,39,46,47$. However, up to 10 different pesticides were detected in three samples of cowpea. Moreover, 99 of the 122 samples with one pesticide residue, 47 of the 109 samples with two pesticide residues, 32 of the 76 samples with three pesticide residues, 14 of the 42 samples with four pesticide residues, 6 of the 50 samples with five pesticide residues, exceeded their MRLs (Fig 2). This could be due to the overuse of mixture pesticides for plants protect, which can lead to major multiresidue regarding food safety ${ }^{39}$. Therefore, effective national food control systems, such as good Agricultural Practices (GAP), which establish a national pesticide monitoring program widely accepted in most countries, are essential to protect the health and safety of domestic consumers.

\section{Comparison of different years}

A total of 574 samples of cowpea (Vigna unguiculata L. Walp) were collected includes 61 samples from 2018, 152 samples from 2019 , 199 samples from 2020, and 162 samples from 2021. The samples from 2018 are relatively small and unrepresentative, so they will not be included in the comparison. A total of 17 pesticides were detected in 2021, 24 pesticides in 2019 and in 2020 (Fig 3a). Moreover, it has a tendency that the detection rate of the same pesticide decreased year by year. In addition, Pesticide residues of azoxystrobin, chlorfenapyr, chlorpyrifos-ethyl, cyhalothrin, cypermethrin, difenoconazole, fenpropathrin, fenvalerate, fipronil, malathion, methamidophos, profenofos, pyridaben, pyrimethanil, and triazophos were detected in 2019 to 2021 , indicating that these pesticides were all used in various years. Compared with 2019, 2 new pesticides were detected in 2020: acephate, coumaphos. In addition, compared with 2020, one new pesticide was detected in 2021: fenitrothion. However, Duan et al. reported that the most important residues of the 433 fresh cowpea samples from Hainan province in 2012 and 2013 were triazophos, carbofuran, isocarbophos, phoxim and omethoate ${ }^{3}$. It showed that the pesticide triazophos was still used now. This might be due to in addition to spraying conventional pesticides, there are also exploratory pesticides used in the annual use of cowpea farmers. As shown in Fig 3b, 10 banned pesticides were detected in 2019, 11 in 2020, and 5 in 2021. Therefore, there is a tendency to decrease the use of banned pesticides. Moreover, 4 pesticides (cyhalothrin, cypermethrin, difenoconazole, and pyridaben) which exceeded their MRLs, were detected in 2019 to 2021, indicating that there may be excessive dosage and times of pesticides used in cowpea.

\section{Comparison by Region}

From 2018 to 2021, a total of 166 samples from Ledong, 136 samples from Lingshui, 115 samples from Sanya, 52 samples from Chengmai, 29 samples from Wanning, 40 samples from Haikou, and 36 sample from Danzhou were collected. The samples from Ledong, Lingshui, and Sanya, which are more relatively and representative than other region, so they will be included in the comparison. A total of 26 pesticides were detected in Ledong, 24 pesticides in Lingshui and 21 pesticides in Sanya (Fig. 4a). In addition, pesticide residues of azoxystrobin, chlordimeform, chlorfenapyr, chlorpyrifos-ethyl, cyfluthrin, cyhalothrin, cypermethrin, dicofol, difenoconazole, fenpropathrin, fenvalerate, fipronil, isazophos, isocarbophos, parathion-methyl, profenofos, pyridaben, pyrimethanil, and triazophos were all detected in Ledong, Lingshui, and Sanya, indicating that these pesticides were all used in each production area. As shown in Fig $4 \mathrm{~b}$, 8 banned pesticides (chlordimeform, chlorpyrifos-ethyl, dicofol, fipronil, isazophos, isocarbophos, parathion-methyl, and triazophos) and 3 pesticides (chlorfenapyr, difenoconazole and pyridaben) which exceeded their MRLs, were detected in Ledong, Lingshui, and Sanya. It shows that farmers use prohibited pesticides in these areas, and the source needs to be traced. Therefore, there may be excessive dosage and times of pesticides used in cowpea.

\section{Dietary exposure risk assessment}

Dietary exposure was used to assess the possible exposure routes and dose levels and to clarify the actual and expected exposure levels and possible harm caused to sensitive groups. The chronic hazard quotients of different populations calculated based on average pesticide residues were listed in shown in Table $3^{48}$. The chronic hazard quotient of all pesticides detected in cowpea was less than $100 \%$, indicating that the pesticide residues in Hainan cowpea had little contribution to the risk of chronic dietary exposure. The magnitude of chronic hazard quotient in different groups of the same gender was consistent with ( $2 \sim 7$ years old $)>(8 \sim 12$ years old $)>(13 \sim 19$ years old $) \geq(>65$ years old $) \geq(20 \sim 50$ years old $) \geq(51 \sim 65$ years old $)$, which was caused by the weight difference of different groups and cowpea intake. The magnitude of the chronic hazard quotient of the same population of different genders was consistent with that of female $\geq$ male, because female's weight and daily intake was lower than that of male. The analysis revealed that dietary exposure gradually decreased with age, and children (2 7 years old) had the highest dietary exposure. In addition, the dietary exposure of female was slightly higher than that of male of the same age group. A similar phenomenon has also been observed in previous studies ${ }^{1,46,47}$. In fact, unlike foreigners, Chinese people are used to stir-frying 
vegetables before eating, which could reduce the risk ${ }^{3}$. Similarly, it also has been reported that blanching ( 5 min) followed by stir-frying ( 3 min) was recommended to the citizens as the safest household processing way to cowpea ${ }^{49}$. Therefore, we suggested that the cowpeas should be blanching or stirfrying before eating to reduce the risk.

\section{Conclusions}

A QuEChERS-GC-MS/MS method for simultaneous determination of 35 pesticides in cowpea was successfully validated. It showed satisfactory recoveries and precision (70-120\%, RSD below $20 \%$ ) at 50,100 and $250 \mu \mathrm{g} / \mathrm{kg}$ for 35 pesticides. In addition, the limit of quantification can meet the detection requirements of maximum residue limits of 35 pesticides in cowpea of Eu or other countries. A total of 574 samples of cowpea from Hainan province of China were analyzed, and $80.8 \%$ of them were positive. According to the actual survey in each producing area, the possibility of active use of restricted pesticides in production is low. However, 12 kinds of restricted pesticides were detected in verification analysis, indicating that farmers use restricted pesticides and their sources need to be traced. Residues in $30.1 \%$ of the samples for ten pesticides exceeded their MRLs and twelve banned pesticides. In addition, the forbidden pesticide triazophos was detected through different years and regions. From the perspective of pesticide MRLS and dietary risk, the pesticide residue level of cowpea in Hainan province is not high, and the chronic dietary risk of pesticides in different genders and ages was very low (<3\%), within the acceptable range $(<100 \%)$, providing technical support for human health protection.

\section{Declarations}

\section{Acknowledgments}

This work was supported in part by the Hainan Natural Science Foundation for High-level Talents (No. 320RC679), the National Agricultural Product Quality Safety Risk Assessment Program of China (No. GJFP201900605), the Research Project of Hainan Academician Innovation Platform (No. YSPTZX202021), and Fundamental Research Funds of Chinese Academy of Tropical Agricultural Sciences (No. 1251632021002).

\section{Author contributions}

Q. Z. designed the experiments and wrote the manuscript text, and Q. Z., C. M., Y. D., X. W., D. L. and J. L. performed experiments.

\section{References}

1. Huan, Z. B., Xu, Z., Luo, J. H. \& Xie, D. F. Monitoring and exposure assessment of pesticide residues in cowpea (vigna unguiculata I. walp) from five provinces of southern china. Regu.I Toxicol. Pharmacol, 81, 260-267 (2016).

2. Statistical Bureau of Hainan Province, Survey Office of National Bureau of Statistics in Hainan. Hainan Statistical Yearbook. Beijing: China Statistical Press (2020) [Chinese edition].

3. Duan, Y., Guan N., Li, P. P., Li, J. G. \& Luo J. H. Monitoring and dietary exposure assessment of pesticide residues in cowpea (Vigna unguiculata L. Walp) in Hainan, China. Food Control, 59, 250-255 (2016).

4. Seydou, D. et al. Push-pull strategy combined with net houses for controlling cowpea insect pests and enhancing crop yields. Crop Prot. 141,105480 (2020).

5. Ozcan, C. \& Balkan, S. Multi-residue determination of organochlorine pesticides in vegetables in Kirklareli, Turkey by gas chromatography-mass spectrometry. J. Anal. Chem. 72, 761-769 (2017).

6. Zhao, L. M., Szakas T., Churley M. \& Lucas D. Multi-class multi-residue analysis of pesticides in edible oils by gas chromatography-tandem mass spectrometry using liquid-liquid extraction and enhanced matrix removal lipid cartridge cleanup. J. Chromatogr. A 1584, 1-12 (2019).

7. Hejji, L., Azzouz, A., Colón, L. P., Souhail, B. \& Ballesteros, E. A multi-residue method for determining twenty-four endocrine disrupting chemicals in vegetables and fruits using ultrasound-assisted solid-liquid extraction and continuous solid-phase extraction. Chemosphere 263, 128158 (2020).

8. da Silva Sousa, J., do Nascimento, H. O., de Oliveira Gomes, H. \& do Nascimento, R. F. Pesticide residues in groundwater and surface water: recent advances in solid-phase extraction and solid-phase microextraction sample preparation methods for multiclass analysis by gas chromatography-mass spectrometry. Microchem. J. 168, 106359 (2021).

9. Zhou, J. et al. Study on environmental behaviour of fluopyram in different banana planting soil. Sci. Rep. 11, 15346 (2021).

10. Paepe, E. D. et al. Ultra-high-performance liquid chromatography coupled to quadrupole orbitrap high-resolution mass spectrometry for multi-residue screening of pesticides, (veterinary) drugs and mycotoxins in edible insects. Food Chem. 293, 187-196 (2019).

11. Choi, S. Y. \& Kang, H. S. Multi-residue determination of sulfonamides, dapsone, ormethoprim, and trimethoprim in fish and shrimp using dispersive solid phase extraction with LC-MS/MS. Food Anal. Method. 14, 1256-1268 (2021).

12. Pang, X. et al. Wide-scope multi-residue analysis of pesticides in beef by ultra-high-performance liquid chromatography coupled with quadrupole time-offlight mass spectrometry[J]. Food Chem. 351, 129345 (2021).

13. Melton, L. M., Taylor, M. J. \& Flynn, E. E. The utilisation of ion chromatography and tandem mass spectrometry (IC- MS/MS) for the multi-residue simultaneous determination of highly polar anionic pesticides in fruit and vegetables. Food Chem. 298, 125028 (2019).

14. Lan, M. et al. Multi-residue detection of pesticides using a sensitive immunochip assay based on nanogold enhancement. Anal. Chim. Acta $938,146-155$ (2016). 
15. Pang, X., Liu, X., Peng, L., Chen, Z. \& Weng, R. A multi-residue electrochemical biosensor based on graphene/chitosan/parathion for sensitive organophosphorus pesticides detection. Chem. Phys. Lett. 767, 138355 (2021).

16. Zhao, G., Zhou, B., Wang, X., Shen, J. \& Zhao, B. Detection of organophosphorus pesticides by nanogold/mercaptomethamidophos multi-residue electrochemical biosensor. Food Chem. 354, 29511 (2021).

17. Xu, F. et al. Simultaneous determination of 25 pesticides in Zizania latifolia by dispersive solid-phase extraction and liquid chromatography-tandem mass spectrometry. Sci. Rep. 9, 10031 (2019).

18. Al-Antary, T. M., Alawi, M. A., Masaedeh, M. \& Haddad, N. A. Multi-residue analysis of 405 pesticides in agricultral crops in middle governorates of jordan in 2018 and 2019 using quechers method followed by LC-MS/MS and GC-ECD. Fresen. Environ. Bull. 29, 2534-2539 (2020).

19. Luo, P., Liu, X., Kong, F., Tang, L. \& Li, Y. Multi-residue determination of 325 pesticides in chicken eggs with EMR-Lipid clean-up by UHPLC-MS/MS and GC-MS/MS. Chromatographia 83, 593-599 (2020).

20. Harischandra, N. R. et al. Simultaneous determination of 79 pesticides in pigeonpea grains using GC-MS/MS and LC-MS/MS. Food Chem. $\mathbf{3 4 7}$, 128986 (2021).

21. Wei, Y. et al. Multi-residue Analysis of 34 pesticides in black pepper by QuEChERS with d-SPE vs. d-SLE cleanup. Food Anal. Method. 12, 176-189 (2019).

22. Banno, A. \& Yabuki, Y. Simultaneous analysis of seven neonicotinoid pesticides in agricultural products involving solid-phase extraction and surrogate compensation using liquid chromatography-tandem mass spectrometry. J. Pestic. Sci. 45, 29-38 (2020).

23. Samsul, A., Neha, S., Nusrat, I., Kumar, S. M. \& Jitendra, K. Magnetic solid-phase extraction (MSPE) using magnetite-based core-shell nanoparticles with silica network $\left(\mathrm{SiO}_{2}\right)$ coupled with GC-MS/MS analysis for determination of multiclass pesticides in water. J. AOAC Int. 104, 633-644 (2020).

24. Senosy, I. A., Zhang, X. Z., Lu, Z. H., Guan, X. Y. \& Gbiliy, A. Magnetic metal-organic framework MIL-100 (Fe)/polyethyleneimine composite as an adsorbent for the magnetic solid-phase extraction of fungicides and their determination using HPLC-UV. Microch. Acta 188, 33 (2021).

25. Navickiene, S., Santos, L. F. S. \& dos Reis Silva, A. Use of magnesium silicate as a new type of adsorbent for dispersive solid-phase extraction cleanup of the quick, cheap, effective, rugged, and safe method for pesticides during analysis of lager beer by gas chromatography-tandem mass spectrometry. $J$. AOAC Int. 102, 619-624 (2019).

26. Singh, M., Srivastava, A., Sharma, Y. K., Singh, S. \& Singh, S. P. CVD grown carbon nanofibers: an efficient DSPE sorbent for cleanup of multi-class pesticide residue in high fat and low water commodities by QuEChERS using GC-ECD. Microch. Acta 187, 490 (2020).

27. Jabali, Y., Millet, M. \& El-Hoz, M. Optimization of a DI-SPME-GC-MS/MS method for multi-residue analysis of pesticides in waters. Microch. J. 147, 83-92 (2019).

28. Han, Y. K. et al. Gas-liquid microextraction coupled with magnetic-assisted dispersive solid-phase extraction clean-up for multi-residue pesticide analysis in fatty foods of animal origin. LWT- Food Sc. Technol. 137, 110448 (2020).

29. Hakme, E., \& Poulsen, M. E. Evaluation of the automated micro-solid phase extraction clean-up system for the analysis of pesticide residues in cereals by gas chromatography-orbitrap mass spectrometry. J. Chromatogr. A 1652, 462384 (2021).

30. Kinross, A. D., Hageman, K. J., Doucette, W. J. \& Foster, A. L. Comparison of accelerated solvent extraction (ASE) and energized dispersive guided extraction (EDGE) for the analysis of pesticides in leaves[J]. J. Chromatogr. A 1627, 461414 (2020).

31. Mousavi, M. M., Javanmardi, F., Andishmand, H., Momeni, M. \& Mahmoudpour, M. Vortex and ultrasound-assisted surfactant-enhanced emulsification microextraction for the determination of pesticide residues in honey using gas chromatography-mass spectrometry. J. Anal. Chem. 75, 1153-1161 (2020).

32. Li, M. et al. Chemometric-assisted QuEChERS extraction method for post-harvest pesticide determination in fruits and vegetables. Sci. Rep. 7, 42489 (2017).

33. Tian, F. et al. Method development and validation of ten pyrethroid insecticides in edible mushrooms by modified QuEChERS and gas chromatographytandem mass spectrometry. Sci. Rep. 10, 7042 (2020).

34. Beguiristain, I., Alongi, A., Rúbies, A. \& Granados, M. Analysis of corticosteroids in samples of animal origin using QuEChERS and ultrahigh-performance liquid chromatography coupled to high-resolution mass spectrometry. Anal. Bioanal. Chem. 411, 449-457 (2018).

35. Reichert, B. et al. Pesticide residues determination in common bean using an optimized QuEChERS approach followed by solvent exchange and GCMS/MS analysis. J. Sci. Food and Agr. 100, 2425-2434 (2020)

36. Acosta-Dacal, A., Rial-Berriel, C., Díaz-Díaz, R., del Mar Bernal Suárez, M. \& Luzardo, O. P. Optimization and validation of a QuEChERS-based method for the simultaneous environmental monitoring of 218 pesticide residues in clay loam soil. Sci. Total Environ. 753, 142015 (2021).

37. Sampling specification for pesticide residue detection in vegetables: NY/T 762-2004, China Agriculture Press, Beijing, (2004) [Chinese edition].

38. Guidance SANTE/12682/2019 - Guidance document on analytical quality control and method validation procedures for pesticides residues analysis in food and feed. European Commission. Directorate General for Health and Food Safety (2019).

39. Li, Z. X. A monitoring survey and dietary risk assessment for pesticide residues on peaches in China. Regul. Toxicol. Pharm. 97, 97, 152-162 (2018).

40. Rutkowska, E., Łozowicka, B. \& Kaczyński, P. Modification of multiresidue QuEChERS protocol to minimize matrix effect and improve recoveries for determination of pesticide residues in dried herbs followed by GC-MS/MS. Food Anal. Method. 11, 709-724 (2017).

41. Krynitsky, A., Wong, J., Zhang, K. \& Safarpour, H. Important considerations regarding matrix effects when developing reliable analytical residue methods using mass spectrometry. LC GC N. Am. 25, 444-451 (2017).

42. National food safety standard-maximum residue limits for pesticides in food: GB 2763-2021, China Agriculture Press, Beijing, (2021) [Chinese edition]. 
43. Ministry of agriculture and rural affairs, the MRLs of pesticide in vegetable routine monitoring in 2015.

https://max.book118.com/html/2017/0101/78934222.shtm [Chinese edition].

44. List of pesticides prohibited from production, transportation, storage, sale and use in Hainan special economic zone issued by Hainan department of agriculture and rural affairs. http://law.foodmate.net/show-191602.html [Chinese edition].

45. The maximum residue limits of pesticide in European Commission. https://ec.europa.eu/food/plant/pesticides/eu-pesticides-database/mrls/? event=search.pr

46. Golge, O., Hepsag, F. \& Kabak, B. Health risk assessment of selected pesticide residues in green pepper and cucumber. Regul. Toxicol. Pharm. 121, 51-64 (2018).

47. Chen, R., Xue, X.M., Wang, G. P. \& Wang, J. Z. Determination and dietary intake risk assessment of 14 pesticide residues in apples of China. Food Chem. 351, 129266 (2021).

48. Jin G. S. Chinese people's nutrition and health status survey report no.10. People's Medical Publishing House, Beijing, (2008) [Chinese edition].

49. Huan, Z. B., Xu, Z., Jiang, W. N., Chen, Z. Q. \& Luo, J. H. Effect of Chinese traditional cooking on eight pesticides residue during cowpea processing. Food Chem. 170, 118-122 (2015)

\section{Tables}

Table 1. Retention times, quantitative and qualitative ions pair, collision energies for the tested pesticides in SRM mode.

\begin{tabular}{|llll|}
\hline Fluvalinate I- II & 20.62 (peak1) & $180.8>152.1(22)$ & $250>55.1(16), 250>199.9(18)$ \\
\cline { 2 - 4 } & 20.68 (peak2) & $180.8>152.1(22)$ & $250>55.1(16), 250>199.9(18)$ \\
\hline Isazofos & 11.96 & $118.9>76(18)$ & $161>119(8), 161>146(6)$ \\
\hline Isocarbophos & 14.62 & $121.1>65(14)$ & $136>69(30), 136>108(12)$ \\
\hline Isofenphos-methyl & 14.96 & $199>65(34)$ & $199>121(10), 241.1>121.1(20)$ \\
\hline malathion & 14.13 & $92.8>63(8)$ & $125>79(8), 173.1>99(12)$ \\
\hline Methamidophos & 5.12 & $141>64(18)$ & $141>79(20), 141>94.8(8)$ \\
\hline Omethoate & 9.55 & $110>79(10)$ & $110>80(8), 156>110(8)$ \\
\hline Parathion (ethyl) & 14.51 & $109>81(10)$ & $124.9>97(6), 291>109(12)$ \\
\hline Parathion-methyl & 13.11 & $124.9>47(12)$ & $124.9>79(6), 263>109(12)$ \\
\hline Pendimethalin & 15.07 & $252.1>161(14)$ & $252.1>162(8), 252.1>191.3(8)$ \\
\hline Phorate & 10.63 & $75>47(8)$ & $121>65(8), 262>75(8)$ \\
\hline Profenofos & 16.26 & $296.7>268.9(10)$ & $336.9>266.9(12), 336.9>308.9(8)$ \\
\hline Pyridaben & 19.29 & $147.1>117.1(20)$ & $147.1>119.1(8), 147.1>132.1(12)$ \\
\hline Pyrimethanil & 11.80 & $198.1>117.9(30)$ & $198.1>157.6(18), 198.1>182.9(14)$ \\
\hline Sulfotep & 10.41 & $202>145.9(10)$ & $265.9>145.9(15), 322>202(10)$ \\
\hline Terbufos & 11.52 & $230.9>128.9(22)$ & $230.9>174.9(12), 230.9>203(8)$ \\
\hline Triazophos & 17.17 & $161>105.7(12)$ & $161>134.1(8), 172.1>77.1(25)$ \\
\hline
\end{tabular}

Table 2. Average cowpea intake and body weights of the 10 age/sex groups in China. 


\begin{tabular}{|cccc|}
\hline Age & Sex & Average body weigh $(\mathrm{kg})$ & Average cowpea intake $(\mathrm{g})$ \\
\hline $2 \sim 7$ & - & 17.9 & 10.53 \\
\hline $8 \sim 12$ & - & 33.1 & 15.5 \\
\hline $13 \sim 19$ & $\mathrm{M}^{\mathrm{a}}$ & 56.4 & 18.6 \\
& $\mathrm{~F}^{\mathrm{b}}$ & 50.0 & 19.1 \\
\hline $20 \sim 50$ & $\mathrm{M}^{\mathrm{a}}$ & 63.0 & 21.6 \\
& $\mathrm{~F}^{\mathrm{b}}$ & 56.0 & 20.2 \\
\hline $51 \sim 65$ & $\mathrm{M}^{\mathrm{a}}$ & 65.0 & 22.2 \\
& $\mathrm{~F}^{\mathrm{b}}$ & 58.0 & 20.0 \\
\hline$>65$ & $\mathrm{M}^{\mathrm{a}}$ & 59.5 & 19.4 \\
\cline { 2 - 4 } & $\mathrm{F}^{\mathrm{b}}$ & 52.0 & 18.0 \\
\hline
\end{tabular}

Noteð ${ }^{a}$ male, ${ }^{b}$ female.

Table 3. Chronic dietary exposure risk (\%ADI) of pesticide residue in Hainan cowpea samples among different subgroups based on average concentration.

Note: a Maximum residue limits: ${ }^{\mathrm{a} 1}$ The pesticide is banned on legumes in China, ${ }^{\mathrm{a} 2}$ The Chinese national standard GB/T 2763-2021, ${ }^{\mathrm{a} 3}$ The maximum residue limits of pesticide in vegetable routine monitoring in $2015,{ }^{a 4}$ The maximum residue limits of pesticide in European Commission (https://ec.europa.eu/food/plant/pesticides/eu-pesticides-database/mrls/?event=search.pr).

${ }^{\mathrm{b}}$ Acceptable daily intakes (ADIs) was referred to the Chinese national standard GB/T 2763-2021.

Table 4. The results of method accuracy for this study.

Note: ${ }^{a}$ determination coefficient, ${ }^{b}$ limit of detection, ${ }^{C}$ limit of quantification, ${ }^{d}$ relative standard deviations, ${ }^{e}$ Matrix effect.

\section{Figures}


Pesticide

Chronic dietary exposure risk (\%ADI) of different subgroups (age)

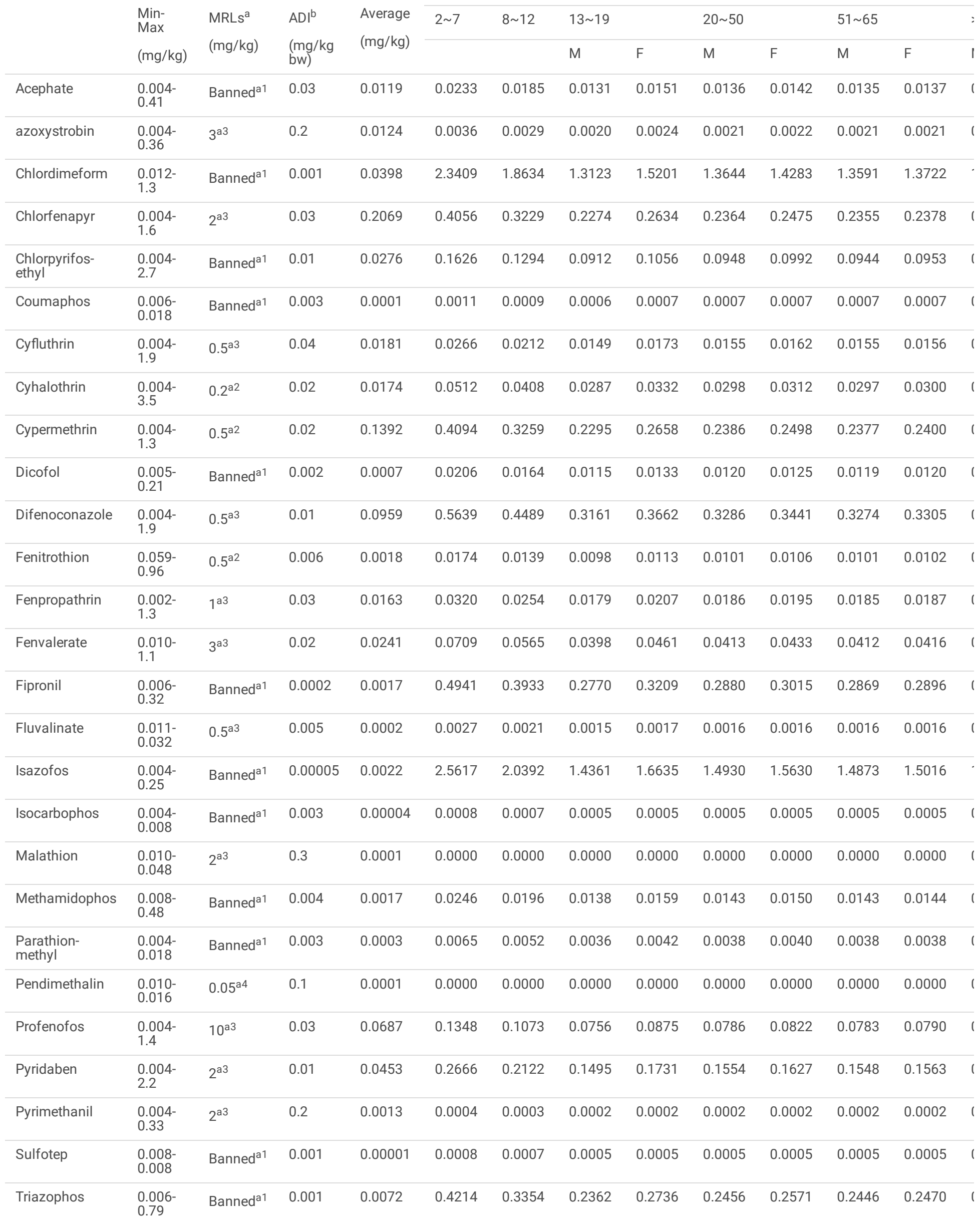




\begin{tabular}{|c|c|c|c|c|c|c|c|}
\hline \multirow[t]{2}{*}{ Pesticide } & \multirow[t]{2}{*}{ Calibration curve equations } & \multirow[t]{2}{*}{$\mathrm{R}^{2 \mathrm{a}}$} & \multirow{2}{*}{$\begin{array}{l}\mathrm{LOD}^{\mathrm{b}} / \mathrm{LOQ}^{\mathrm{c}} \\
(\mu \mathrm{g} / \mathrm{kg})\end{array}$} & \multicolumn{3}{|c|}{ Average recovereries $(\%) \pm\left(R S D s^{d} \rrbracket \% \llbracket n=5\right)$} & \multirow{2}{*}{$\begin{array}{l}\mathrm{ME}^{\mathrm{e}} \\
(\%)\end{array}$} \\
\hline & & & & $50 \mu \mathrm{g} / \mathrm{kg}$ & $100 \mu \mathrm{g} / \mathrm{kg}$ & $250 \mu \mathrm{g} / \mathrm{kg}$ & \\
\hline Acephate & $Y=5.212 e^{4} X-1.546 e^{3}$ & 0.9999 & $2.5 / 8.0$ & $92.3 \pm 6.2$ & $86.1 \pm 2.7$ & $92.8 \pm 3.1$ & 57.8 \\
\hline azoxystrobin & $Y=1.705 e^{4} X+3.256 e^{4}$ & 0.9999 & $0.3 / 1.0$ & $102.6 \pm 4.2$ & $113.6 \pm 4.2$ & $113.8 \pm 1.6$ & 60.4 \\
\hline Chlordimeform & $Y=3.537 e^{4} X+2.574 e^{5}$ & 0.9999 & $2.0 / 6.0$ & $96.2 \pm 7.8$ & $92.9 \pm 8.4$ & $110.5 \pm 1.8$ & 23.03 \\
\hline Chlorfenapyr & $Y=7.664 e^{3} X+3.752 e^{4}$ & 0.9999 & $2.0 / 6.0$ & $88.0 \pm 3.6$ & $96.8 \pm 4.4$ & $102.3 \pm 0.5$ & 35.5 \\
\hline Chlorpyrifos-ethyl & $Y=1.744 e^{5} X+1.16 e^{5}$ & 1.0000 & $0.3 / 1.0$ & $114.0 \pm 9.7$ & $109.1 \pm 2.6$ & $103.7 \pm 1.1$ & 38.2 \\
\hline Coumaphos & $Y=1.695 e^{4} X+2.614 e^{4}$ & 0.9998 & $1.5 / 5.0$ & $96.2 \pm 3.9$ & $99.3 \pm 5.1$ & $102.2 \pm 2.5$ & 75.88 \\
\hline Cyfluthrin I-IVa & $Y=7.225 e^{4} X-4.985 e^{4}$ & 0.9999 & $1.5 / 5.0$ & $96.6 \pm 4.9$ & $96.1 \pm 1.4$ & $96.0 \pm 1.3$ & 37.49 \\
\hline Cyhalothrin I-Ila & $Y=2.142 e^{5} X+4.511 e^{5}$ & 0.9998 & $1.5 / 5.0$ & $77.6 \pm 1.8$ & $90.8 \pm 2.2$ & $101.9 \pm 1.2$ & 66.8 \\
\hline Cypermethrin I-IVa & $Y=9.391 e^{4} X+6.153 e^{5}$ & 0.9999 & $0.6 / 2.0$ & $98.0 \pm 9.8$ & $99.9 \pm 6.5$ & $89.3 \pm 2.7$ & 41.88 \\
\hline Dicofol & $Y=6.647 e^{3} X+1.245 e^{3}$ & 0.9999 & $1.5 / 2.5$ & $110.5 \pm 4.6$ & $107.9 \pm 4.9$ & $101.3 \pm 3.0$ & 48.47 \\
\hline Difenoconazole I-Ila & $Y=1.755 e^{5} X+2.396 e^{5}$ & 0.9999 & $0.3 / 1.0$ & $90.8 \pm 2.5$ & $100.3 \pm 3.3$ & $112.6 \pm 2.3$ & 65.57 \\
\hline Dimethoate & $Y=3.456 e^{4} X-8.066 e^{4}$ & 0.9999 & $1.5 / 5.0$ & $112.2 \pm 5.8$ & $114.4 \pm 3.4$ & $97.9 \pm 1.5$ & 64.1 \\
\hline Endosulfan I-Ila & $Y=2.1338 e^{4} X-2.136 e^{3}$ & 0.9999 & $1.5 / 5.0$ & $105.9 \pm 1.2$ & $97.0 \pm 0.9$ & $96.8 \pm 0.4$ & 19.71 \\
\hline Ethoprophos & $Y=7.035 e^{4} X-6.293 e^{4}$ & 0.9999 & $0.3 / 1.0$ & $102.9 \pm 0.9$ & $106.5 \pm 1.2$ & $99.7 \pm 1.6$ & 54.34 \\
\hline Fenitrothion & $Y=4.158 e^{4} X+5.831 e^{5}$ & 0.9999 & $2.0 / 6.0$ & $93.6 \pm 6.9$ & $104.0 \pm 6.1$ & $101.2 \pm 1.7$ & 81.81 \\
\hline Fenpropathrin & $Y=1.811 e^{5} X+4.155 e^{5}$ & 0.9991 & $2.0 / 6.0$ & $94.5 \pm 1.9$ & $95.2 \pm 1.6$ & $111.3 \pm 2.1$ & 28.83 \\
\hline Fenvalerate & $Y=1.156 e^{4} X+2.08 e^{4}$ & 1.0000 & $1.0 / 3.0$ & $103.1 \pm 7.1$ & $108.5 \pm 6.9$ & $91.7 \pm 2.4$ & 42.07 \\
\hline Fipronil & $Y=6.462 e^{4} X-2.177 e^{5}$ & 0.9995 & $1.0 / 3.0$ & $101.4 \pm 1.0$ & $94.2 \pm 1.2$ & $95.6 \pm 0.7$ & 63.22 \\
\hline Fluvalinate I-Ila & $Y=5.759 e^{4} X-2.698 e^{5}$ & 0.9990 & $1.5 / 5.0$ & $98.1 \pm 9.5$ & $84.5 \pm 1.4$ & $83.2 \pm 4.7$ & 74.62 \\
\hline Isazofos & $Y=3.008 e^{5} X-2.941 e^{5}$ & 1.0000 & $1.0 / 3.0$ & $107.2 \pm 1.4$ & $102.7 \pm 2.1$ & $108.5 \pm 0.9$ & 11.08 \\
\hline Isocarbophos & $Y=6.758 e^{4} X-1.244 e^{5}$ & 0.9999 & $0.6 / 2.0$ & $108.5 \pm 0.9$ & $102.7 \pm 4.9$ & $99.3 \pm 1.2$ & 49.25 \\
\hline |sofenphos-methyl & $Y=1.054 e^{5} X-1.818 e^{5}$ & 0.9999 & $0.6 / 2.0$ & $104.3 \pm 3.8$ & $98.3 \pm 3.2$ & $96.3 \pm 0.9$ & 43.15 \\
\hline malathion & $Y=1.378 e^{5} X+5.087 e^{6}$ & 0.9997 & $0.6 / 2.0$ & $91.4 \pm 7.4$ & $91.0 \pm 6.6$ & $96.8 \pm 4.3$ & 43.77 \\
\hline Methamidophos & $Y=1.278 e^{4} X-2.398 e^{4}$ & 0.9998 & $1.0 / 3.0$ & $94.6 \pm 2.1$ & $91.8 \pm 1.7$ & $86.3 \pm 2.2$ & 35.38 \\
\hline Omethoate & $Y=2.033 e^{5} X+5.787 e^{5}$ & 0.9995 & $1.5 / 5.0$ & $110.1 \pm 9.5$ & $95.5 \pm 2.9$ & $93.2 \pm 1.6$ & -68.57 \\
\hline Parathion (ethyl) & $Y=5.402 e^{4} X-1.72 e^{5}$ & 0.9997 & $1.0 / 3.0$ & $101.7 \pm 3.9$ & $97.1 \pm 2.6$ & $94.5 \pm 1.2$ & 76.31 \\
\hline Parathion-methyl & $Y=8.471 e^{4} X-1.55 e^{5}$ & 0.9999 & $1.0 / 3.0$ & $99.8 \pm 3.2$ & $99.1 \pm 1.5$ & $98.2 \pm 1.4$ & 75.82 \\
\hline Pendimethalin & $Y=3.94 e^{4} X-1.602 e^{5}$ & 0.9993 & $2.5 / 7.5$ & $97.9 \pm 3.0$ & $91.9 \pm 1.9$ & $87.5 \pm 1.1$ & 45.01 \\
\hline Phorate & $Y=3.409 e^{4} X-1.537 e^{4}$ & 1.0000 & $1.0 / 3.0$ & $116.5 \pm 2.3$ & $104.7 \pm 0.7$ & $100.8 \pm 1.6$ & 36.36 \\
\hline Profenofos & $Y=9.018 e^{4} X+2.566 e^{4}$ & 1.0000 & $1.0 / 3.0$ & $108.4 \pm 1.9$ & $103.3 \pm 2.0$ & $100.1 \pm 1.2$ & 81.08 \\
\hline Pyridaben & $Y=2.989 e^{5} X+7.01 e^{5}$ & 0.9998 & $0.5 / 1.5$ & $85.5 \pm 3.7$ & $96.4 \pm 2.1$ & $103.8 \pm 1.9$ & 55.19 \\
\hline Pyrimethanil & $Y=9.968 e^{4} X-9.818 e^{3}$ & 1.0000 & $0.3 / 1.0$ & $100.7 \pm 0.8$ & $96.9 \pm 1.3$ & $94.9 \pm 0.7$ & 35.95 \\
\hline Sulfotep & $Y=7.091 e^{4} X-4.793^{3}$ & 1.0000 & $2.0 / 6.0$ & $108.7 \pm 2.2$ & $101.4 \pm 1.1$ & $101.2 \pm 1.7$ & 37.8 \\
\hline Terbufos & $Y=1.85 e^{5} X+7.66 e^{2}$ & 1.0000 & $1.0 / 3.0$ & $114.7 \pm 2.2$ & $105.2 \pm 1.8$ & $101.9 \pm 1.3$ & 34.25 \\
\hline Triazophos & $Y=2.091 e^{4} X+2.213 e^{4}$ & 0.9999 & $0.3 / 1.0$ & $103.6 \pm 3.6$ & $96.6 \pm 2.9$ & $96.2 \pm 0.9$ & 60.72 \\
\hline
\end{tabular}




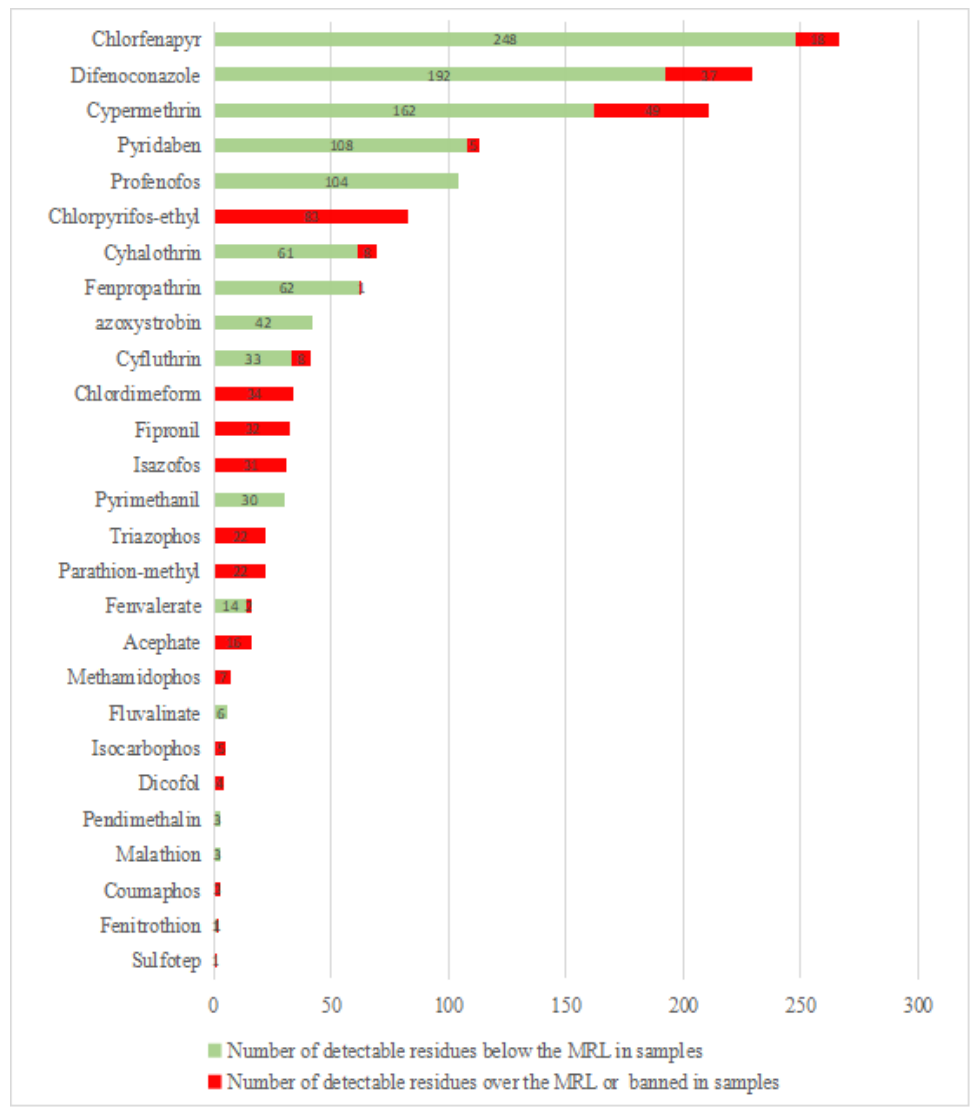

Figure 1

The pesticide in the detected samples.

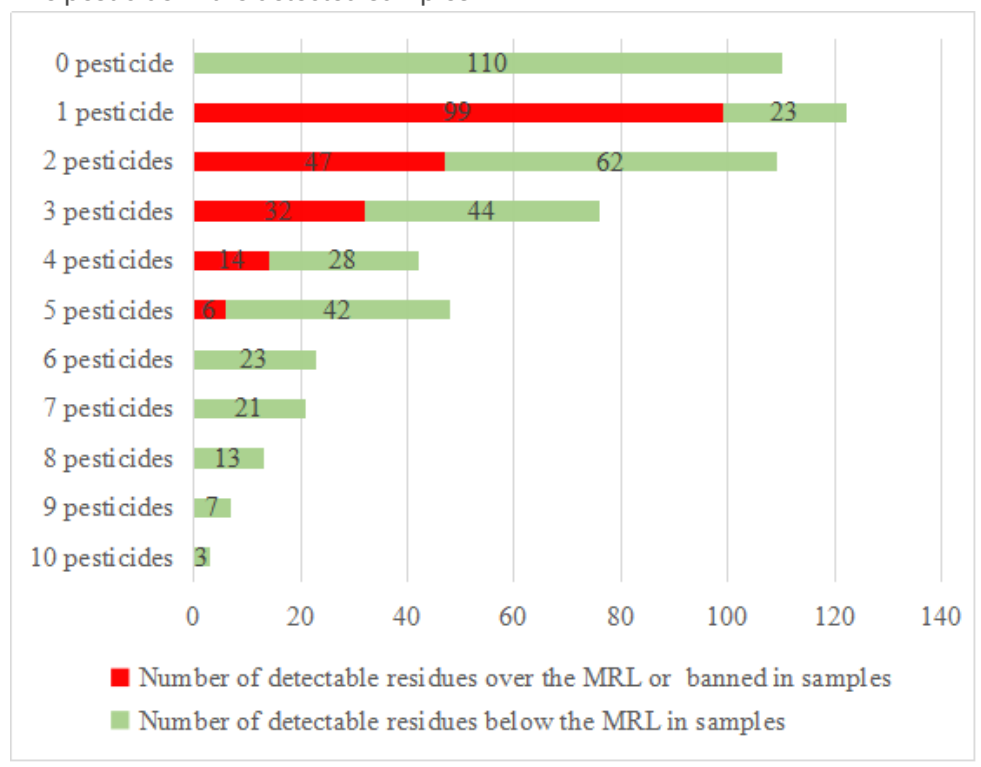

Figure 2

Number of detectable residues in individual cowpea samples. 


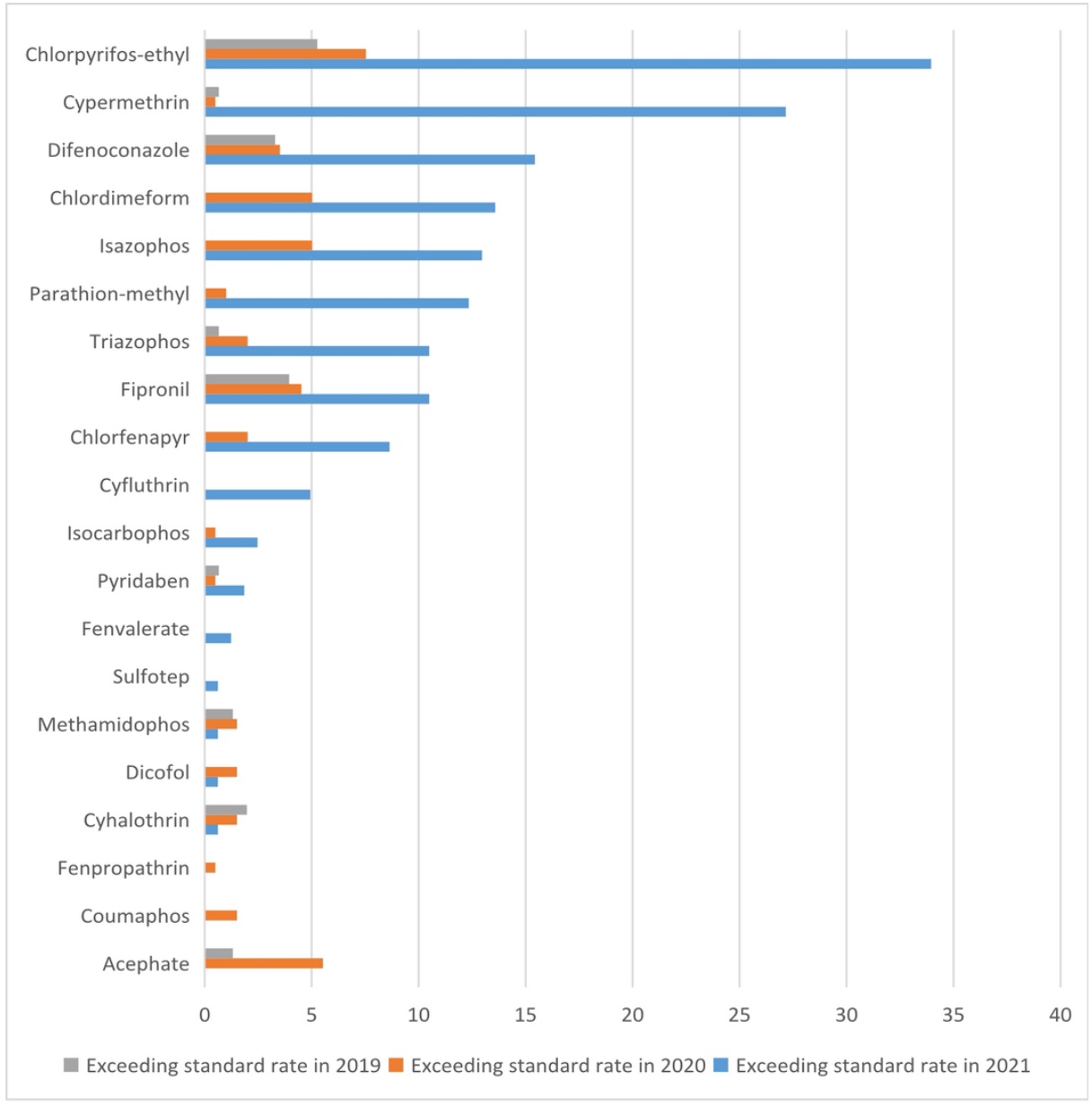

\section{Figure 3}

Comparison of pesticide residue detection rate and over standard rate of cowpea in different years. 


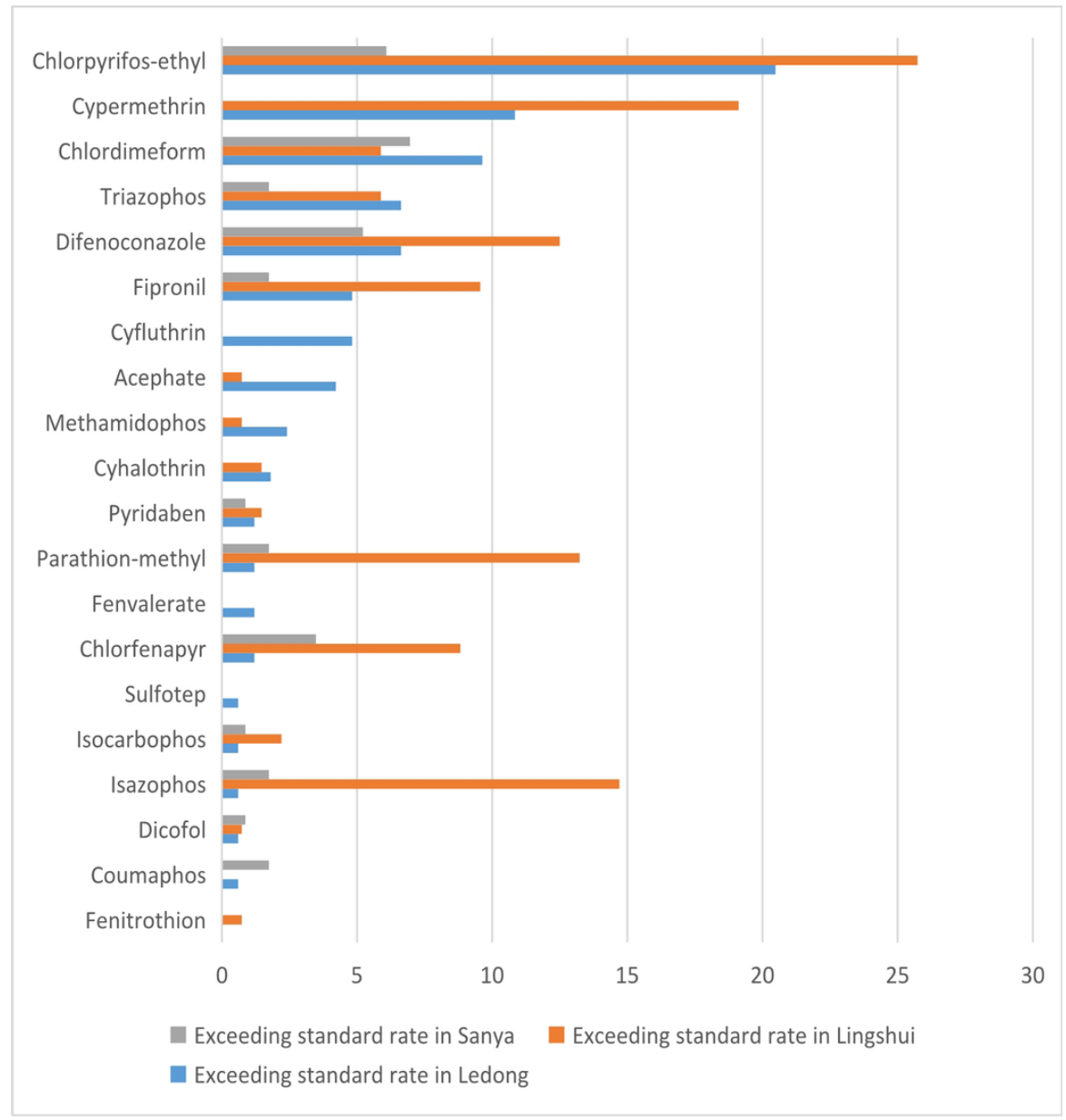

\section{Figure 4}

Comparison of pesticide residue detection rate and over standard rate of cowpea in different areas. 